\title{
Le statut juridique des travailleurs saisonniers en République de Serbie
}

\author{
Ljubinka Kovačević
}

\section{(2) OpenEdition}

1 Journals

\section{Édition électronique}

URL : https://journals.openedition.org/rdctss/756

DOI : $10.4000 /$ rdctss. 756

ISSN : 2262-9815

Traduction(s) :

Legal Position of Seasonal Workers in the Republic of Serbia - URL : https://journals.openedition.org/ rdctss/2776 [en]

Éditeur

Centre de droit comparé du travail et de la sécurité sociale

Édition imprimée

Date de publication : 1 avril 2021

Pagination : 188-193

ISSN : $2117-4350$

\section{Référence électronique}

Ljubinka Kovačević, "Le statut juridique des travailleurs saisonniers en République de Serbie », Revue de droit comparé du travail et de la sécurité sociale [En ligne], 1 | 2021, mis en ligne le 01 avril 2021, consulté le 03 avril 2022. URL : http://journals.openedition.org/rdctss/756 ; DOI : https://doi.org/ $10.4000 /$ rdctss.756

\section{(c)}

Revue de droit comparé du travail et de la sécurité sociale est mise à disposition selon les termes de la Licence Creative Commons Attribution - Pas d'Utilisation Commerciale - Pas de Modification 4.0 International. 


\section{LJUBINKA KOVAČEVIĆ}

Université de BeLgRADE

\section{LE STATUT JURIDIQUE DES TRAVAILLEURS SAISONNIERS EN RÉPUBLIQUE DE SERBIE}

En République de Serbie, le droit offre deux possibilités à l'employeur ayant besoin de recourir au travail saisonnier : embaucher un travailleur soit sous contrat de travail à durée déterminée, soit sous contrat de travail temporaire et occasionnel. Dans le premier cas, les règles générales relatives à la relation de travail s'appliquent aux travailleurs saisonniers, tandis que dans le cas du travail sur la base d'un contrat de travail temporaire et occasionnel, deux situations juridiques peuvent se présenter.

Conformément à la Loi sur le travail, les travailleurs saisonniers peuvent être embauchés en vertu d'un contrat de travail temporaire et occasionnel conclu sous forme écrite, pour un maximum de 120 jours ouvrés par année civile, avec une assurance obligatoire couvrant les risques : maladie, accidents du travail, maladies professionnelles, vieillesse, invalidité, dommages corporels, décès de l'aidant familial et chômage.

Un deuxième régime est établi par la Loi sur l'emploi simplifié des travailleurs saisonniers dans certaines activités (la LESTS) ${ }^{1}$, qui prévoit que les travailleurs saisonniers peuvent être embauchés sous contrat de travail temporaire et occasionnel pour lequel la forme écrite n'est pas requise. La sécurité sociale obligatoire comprend alors seulement l'assurance maladie en cas d'accident du travail et de maladie professionnelle, ainsi que les droits appropriés à l'assurance retraite et à l'invalidité si un accident du travail ou une maladie professionnelle entraînait l'invalidité ou le dommage corporel du travailleur. La LESTS réglemente l'engagement des travailleurs uniquement dans le secteur de l'agriculture, de la sylviculture et de la pêche, et définit l'employeur comme la personne morale ou physique (entrepreneur et titulaire ou le membre d'une exploitation familiale engagée dans la production agricole).

Les travailleurs engagés conformément à la LESTS ne bénéficient pas de droits découlant de la relation de travail. Les seules exceptions sont le droit à la protection de la santé et à la sécurité au travail, le droit à la protection contre les discriminations, le droit à la protection contre le harcèlement au travail et le droit à la protection des lanceurs d'alerte contre les représailles de l'employeur, sachant que l'application des lois spéciales qui régissent ces questions est étendue à certaines catégories de personnes engagées sous contrat de droit civil et de droit des affaires, dont le contrat de travail temporaire et occasionnel ${ }^{2}$.

1 J. O. RS, $n^{\circ} 50 / 18$

2 Loi sur la sécurité et la santé au travail (J.O. RS, n¹01/05, 91/15 et 113/17), article 4, point 1 ; Loi sur l'interdiction des discriminations (J.O. RS, $n^{\circ} 22 / 09$ ), article 16, paragraphe 2; Loi sur la prévention du harcèlement au travail (J.O. RS, n 36/10), article 2 ; Loi sur la protection des lanceurs d'alerte (J.O.RS, n¹28/14), article 2, alinéas 2 et 5. 
En outre, il faut garder à l'esprit que, conformément à la LESTS, les travailleurs saisonniers bénéficient de certaines dispositions concernant leurs conditions de travail. II s'agit d'abord de l'obligation de l'employeur d'informer les travailleurs saisonniers, avant le début du travail, des tâches à accomplir, du lieu de travail, de la durée prévue de l'engagement, des règles relatives à la santé et à la sécurité au travail, de la durée journalière et hebdomadaire de travail, du temps de pause et du repos journalier et hebdomadaire, ainsi que du montant et des délais de versement de la rémunération ${ }^{3}$. Celle-ci est calculée à l'heure travaillée; elle ne peut être inférieure au montant du salaire horaire minimum valable le jour du paiement et est versée à la fin de la journée de travail, ou éventuellement selon une autre périodicité convenue.

En revanche, la durée de travail quotidienne des travailleurs saisonniers ne doit pas excéder 12 heures par jour, ce qui est assez élevé au regard de la santé et de la sécurité des travailleurs, ainsi que de leur vie familiale et privée. En outre, ces travailleurs ont droit à une pause d'au moins 30 minutes s'ils travaillent huit heures par jour ou plus, ce qui est également nettement moins favorable que le régime applicable aux salariés. Quant à la cessation de l'emploi, l'employeur a le droit d'initier la rupture de la relation de travail lorsque le besoin n'existe plus ou si le travailleur n'exécute pas sa prestation de travail de manière satisfaisante.

L'employeur peut engager des travailleurs saisonniers pour un maximum de 180 jours au cours d'une année civile. Le décompte débute à partir de la déclaration initiale d'embauche d'un travailleur saisonnier effectuée via le site Internet de l'administration fiscale, jusqu'à la déclaration de radiation du dernier travailleur saisonnier sur ce même site, à l'exclusion des jours non travaillés. En ce qui concerne la durée de l'engagement d'un travailleur saisonnier en particulier, l'employeur peut embaucher une même personne pour un maximum de 120 jours ouvrés par année civile. Ce maximum de jours comprend non seulement les jours de travail effectués en vertu de la LESTS et en vertu d'un contrat de travail temporaire et occasionnel conformément à la Loi sur le travail ${ }^{4}$.

L'employeur est tenu de fournir une attestation écrite au travailleur saisonnier, à sa demande, contenant les informations sur les conditions de travail, ainsi que sur toute éventuelle modification des conditions de travail, dans les deux mois au plus tard à compter du début de l'exécution du travail. Cela permet aux travailleurs de bien comprendre leur propre situation juridique et, en cas de conflit, de prouver plus facilement les conditions de travail préalablement convenues. Par ailleurs, l'obligation d'information contribue également à la suppression du « travail au noir », car l'inexistence de document écrit par l'employeur sur les conditions de travail peut servir à l'inspection du travail d'indice de violations des droits des travailleurs saisonniers. Une telle solution est conforme aux dispositions de la Directive (UE) 2019/1152 du Parlement européen et du Conseil du 20 juin 2019 relative à des

3 Règlement sur le contenu de l'attestation de travail d'un travail saisonnier (J.O. RS, n67/18).

4 Par exemple, en 2019, un grand nombre d'employeurs ont embauché des travailleurs saisonniers pour une période d'environ un mois, en moyenne de 15 à 21 jours par an. Voir M. Andjelković Đoković, I. Šmigić, M. Jovanović, Emploi saisonnier dans l'agriculture: analyse des effets de la réforme, Alliance nationale pour le développement économique local, Belgrade, 2020, p. 60. 
conditions de travail transparentes et prévisibles dans l'Union européenne. Mais à l'instar de cette directive, il serait nécessaire d'introduire dans le système juridique serbe des règles pour la protection des droits des travailleurs en tenant compte des travailleurs qui estiment que l'employeur a manqué à son obligation de fournir une déclaration écrite. Cela semble particulièrement important compte tenu de la difficulté des travailleurs à prouver devant les tribunaux le préjudice subi en raison d'un manquement de l'employeur à son devoir de les informer correctement et pleinement sur les conditions de travail.

D'un autre côté, la Directive 2019/1152 établit également un catalogue de droits minimaux pour tous les travailleurs. Il serait extrêmement pertinent d'harmoniser la LESTS avec les dispositions de cette directive, dans la partie relative à la reconnaissance du « droit à la formation » que l'employeur est tenu de fournir aux travailleurs, en la prenant à sa charge et en la considérant comme du temps travaillé car effectuée pendant l'horaire de travail. En outre, en vertu de la directive, les travailleurs ont le "droit à la prévisibilité minimale du travail », c'est-à-dire d'être informés à l'avance de nouvelles tâches à exécuter, avec le droit de refuser leur exécution en dehors de l'horaire de travail convenu (de référence) et d'être protégés contre un éventuel traitement défavorable de l'employeur pour cette raison. La Directive confirme aussi le droit des travailleurs à une compensation en cas de résiliation du contrat de travail par l'employeur avant l'expiration de la période de travail convenue ; une solution qui devrait également être prévue dans la LESTS.

Outre la simplification de la procédure d'embauche, la LESTS prévoit également une « déclaration simplifiée des travailleurs saisonniers auprès de l'Administration fiscale et du Registre central de la sécurité sociale obligatoire ». Les déclarations d'enregistrement et de radiation des travailleurs saisonniers doivent être soumises à l'administration fiscale par voie électronique, pour chaque mois civil. À partir des données figurant dans les déclarations, l'Administration fiscale est tenue ensuite de déposer au Registre central de la sécurité sociale obligatoire la demande d'affiliation à la sécurité sociale obligatoire des travailleurs. Les dispositions de la LESTS précisent également les taux d'imposition et de cotisation, calculés quotidiennement pour chaque travailleur embauché, sur la base de $30 \%$ de la rémunération mensuelle minimale. L'employeur ne paie donc les impôts et les cotisations que pour les jours où le travailleur saisonnier a effectivement été employé, tandis que les impôts et cotisations exigibles lui sont soumis par voie électronique à la fin de chaque mois en cours.

Durant l'exécution du travail, conformément à la LESTS, le travailleur saisonnier n'est pas radié du Registre des demandeurs d'emploi tenu par le Service national de l'emploi. Le versement des allocations de chômage n'est pas non plus suspendu. De même, le fait de percevoir une rémunération pour le travail effectué n'affecte pas l'exercice et l'utilisation du droit à l'aide financière des travailleurs saisonniers, conformément à la réglementation sur la protection sociale. Ces règles ont été introduites dans la LESTS avec l'objectif d'empêcher le travail non déclaré, étant donné qu'un certain nombre de travailleurs saisonniers acceptait de travailler ainsi précisément pour ne pas perdre leurs allocations de chômage ou se trouver hors du système de protection sociale.

Cependant, il semble que cette disposition juridique fasse une distinction injustifiée entre les personnes en état de besoin qui effectuent un travail saisonnier 
sur la base de la LESTS, et tous les autres bénéficiaires de l'aide sociale qui ne peuvent être engagés dans des conditions différentes ${ }^{5}$. Ceci est d'autant plus vrai que l'application de la LESTS permet l'exonération des revenus du travail saisonnier, même dans le cas où les revenus sont relativement élevés, ou lorsque plusieurs membres d'un même foyer effectuent un travail saisonnier concomitamment et pendant plusieurs mois, étant donné que le montant maximum du revenu total annuel des travaux saisonniers au niveau du foyer susceptible d'être exonéré de l'exercice du droit à l'aide sociale financière n'est pas défini.

À cet égard, il convient de garder à l'esprit que l'adoption de la LESTS a été motivée par la nécessité d'empêcher la pratique d'embauche des travailleurs saisonniers issus de l'agriculture dans ce qu'on appelle «la zone grise », étant donné que le travail non déclaré représente 18,2\% de l'emploi en République de Serbie ${ }^{6}$. Cette pratique largement répandue est due : aux coûts élevés du travail générés par un contrat de travail, et par un contrat de travail temporaire et occasionnel conclus conformément à la Loi sur le travail ; à la procédure complexe d'embauche et de déclaration des travailleurs, si bien que les travailleurs acceptent de travailler sans déclaration pour ne pas perdre les aides sociales qu'ils perçoivent ${ }^{7}$. En ce sens, la conséquence positive de l'application de la LESTS se traduit par le fait qu'en 2019, 8,5 fois plus de travailleurs saisonniers (29778 travailleurs saisonniers) ont été officiellement embauchés comparé au nombre total de travailleurs saisonniers enregistrés l'année précédente.

Cependant, le nombre de travailleurs saisonniers formellement engagés en 2019 ne représentait que 37\% du nombre total estimé de travailleurs saisonniers en République de Serbie, le travail non déclaré étant encore très répandu ${ }^{8}$. De ce fait, le ministère du Travail, de l'Emploi, des Anciens combattants et des Affaires sociales, a procédé à la rédaction d'une proposition d'amendement à la LESTS en 2020. II s'agirait d'étendre la possibilité d'emploi simplifié aux travailleurs saisonniers dans

5 En 2019, un peu plus d'un tiers des travailleurs saisonniers agricoles figuraient dans le Registre des demandeurs d'emploi tenu par le Service national de l'emploi et, dans la plupart des cas, il s'agissait de personnes sans travail depuis près de quatre ans. En revanche, seulement $1 \%$ des travailleurs saisonniers bénéficiaient du droit à l'allocation de chômage en même temps qu'ils effectuaient leur travail saisonnier, tandis que 7\% des travailleurs saisonniers travaillaient en même temps sous contrat de travail ou sous contrat de droit civil ou des affaires. Quant aux travailleurs saisonniers bénéficiaires de l'aide sociale financière, ils étaient environ $20 \%$ au cours de la période observée. Voir M. Anđelković, I. Šmigić, M. Jovanović, op. cit., p. 30.

6 I. Đorđević, Ex ante analyse de l'extension du systeme de l'emploi simplifié dans les emplois temporaires et occasionnels, Deutsche Gesellschaft für Internationale Zusammenarbeit (GIZ) GmbH, Belgrade, 2020, p. 8.

7 Ainsi, par exemple, en 2017, le nombre de saisonniers non déclarés dans l'agriculture a été estimé à 70.000 , alors que seulement 3.585 travailleurs saisonniers ont travaillé sous contrat de travail temporaire et occasionnel; un an plus tard, seulement 3.500 travailleurs ont été embauchés sur la base de contrats de travail temporaire et occasionnel, tandis que 65.000 travailleurs saisonniers dans l'agriculture ont travaillé sans base légale et sans déclaration auprès de l'administration fiscale et du registre central de la sécurité sociale obligatoire. M. Anđelković Đoković, I. Šmigić, M. Jovanović, op. cit., p. 58 ; I. Đorđević, op. cit., p. 27.

8 M. Anđelković Đoković, I. Šmigić, M. Jovanović, op. cit., p. 6. 
les secteurs du bâtiment, du tourisme et de la restauration, ainsi qu'aux employés de maison'. L'extension du domaine d'application personnel de la LESTS impliquerait en même temps l'adaptation du système actuel d'emploi simplifié à d'autres activités, tout en respectant leurs particularités.

La proposition du ministère a cependant rencontré des critiques justifiées de la part du public scientifique et professionnel. Cela est dû au fait que la prévention du travail non déclaré est considérée comme la raison principale de l'introduction de nouvelles activités dans le domaine d'application de la LESTS. Bien qu'il s'agisse d'un objectif légitime et important, il ne semble pas acceptable de faire de la réduction du niveau de protection des travailleurs saisonniers un outil pour lutter contre le travail non déclaré. Cela est d'autant plus vrai qu'en République de Serbie, il y a encore à faire pour améliorer le contrôle de l'inspection fiscale et du travail, confrontée à de nombreuses difficultés, dont la plus grave est le nombre insuffisant d'inspecteurs du travail : 250 inspecteurs du travail supervisent actuellement l'application de la législation du travail dans toutes les entreprises enregistrées ou non en Serbie.

Se pose également la question de savoir s'il est justifié d'utiliser le terme saisonnier pour désigner les travaux temporaires et occasionnels dans d'autres activités, vu que contrairement à l'agriculture, elles ne sont pas marquées par des variations saisonnières. Ainsi, par exemple, dans le secteur du bâtiment, il n'est pas possible de parler de travaux saisonniers au sens strict du terme; même dans les pires conditions climatiques, il serait possible d'assurer l'exécution d'un nombre considérable de travaux de construction.

En outre, dans la restauration et le tourisme, il existe un besoin d'engagement des travailleurs sur appel ne signifiant pas nécessairement que ces emplois soient de nature saisonnière. Donner une définition juridique plus précise du travail saisonnier serait nécessaire pour éviter les abus liés au recours simplifié des travailleurs saisonniers même lorsque certaines activités ne sont pas liées à la saison. La liste légale indicative des emplois saisonniers pourrait également y contribuer, telle qu'elle figure dans la LESTS pour l'agriculture, puisque c'est une activité pour laquelle sont précisées les saisons durant lesquelles existe un besoin d'emploi. II en va de même pour le caractère saisonnier de ces emplois, c'est-à-dire du lien étroit entre l'activité et les processus et cycles climatiques et environnementaux, impliquant que certains emplois sont saisonniers (car temporaires ou occasionnels) par leur nature et ne dépendent pas de l'employeur (ni du travailleur).

Enfin, la proposition d'extension du champ d'application de la LESTS ne semble pas acceptable en raison de la crainte que les employeurs souhaitant réduire les coûts de travail (soit pour maintenir la continuité des activités, soit pour maximiser le profit) décident de licencier une partie des salariés et de compenser la nécessité de leur travail par l'embauche simplifiée de travailleurs saisonniers. Ceci n'exclut pas le risque que les travailleurs licenciés soient réembauchés, mais comme travailleurs saisonniers auxquels ne s'appliqueront pas les droits relatifs à la relation de travail.

9 On estime que 11,3\% du nombre total de travailleurs dans l'économie informelle travaillent dans le secteur du bâtiment, tandis que dans la restauration et le tourisme, ce nombre est de 15\%. En outre, on estime que plus de 55000 ménages ont un employé de maison non déclarée. I. Djordjevic, op. cit., p. 15. 


\section{RéPublique de SERbie}

L'élargissement du cercle des travailleurs qui pourraient être engagés pour travailler en dehors de la relation de travail, sans garantie des droits minimaux du travail, ne semble pas acceptable du point de vue du concept de travail décent et de l'exigence de conditions de travail décentes minimales pour tous les travailleurs, quel que soit leur statut.

Cette exigence implique, bien entendu, un catalogue défini de droits individuels fondamentaux, y compris le droit d'être dûment informé et de pouvoir s'organiser en syndicats. 